\title{
Guest editorial: tackling the problems of hematopoietic stem cell aging
}

\author{
Tao Cheng
}

Received: 10 June 2011/ Accepted: 14 June 2011/Published online: 29 June 2011

(C) The Japanese Society of Hematology 2011

Tissue degeneration is a hallmark for the aging of an organism. With the capabilities of self-renewal and differentiation, stem cells are largely responsible for tissue maintenance and regeneration. Therefore, stem cells must be directly involved in organismal aging. In fact, many types of adult stem cells have been reported to be associated with tissue degeneration in a variety of species including human. Research on stem cell aging has been increasingly emphasized in recent years. Since 2009, there have been two international conferences held in Germany on stem cell aging (http://www.ageing-research.de/ index.html). There was a special session on stem cell aging at the ISSCR meeting as well (http://www. isscr.org//AM/Template.cfm?Section=Home). Funding on stem cell aging has also been increased in some countries. For example, the Chinese government recently funded a national key research program on stem cell aging (http:// www.most.gov.cn/tztg/201008/t20100806_78777.htm).

As one of the best studied tissue stem cell types, the hematopoietic stem cell (HSC) gives rise to all the blood and immune cells in the body. Its activity appears to be correlated with mouse lifespan in different genetic backgrounds [1, 2] although earlier studies showed that HSCs could sustain more than a life time [3]. While the question of whether the HSC compartment may become senescent

\section{T. Cheng $(\bowtie)$}

State Key Laboratory of Experimental Hematology, Institute of Hematology, Chinese Academy of Medical Sciences \& Peking Union Medical College, Tianjin 300020,

People's Republic of China

e-mail: chengt@pumc.edu.cn

\section{T. Cheng}

Department of Radiation Oncology, University of Pittsburgh School of Medicine, Pittsburgh, PA 15213, USA in vivo under homeostatic conditions may still be subject to debate, it is clear that the functions of HSCs do change during the lifetime of an organism. For example, increased cycling and biased differentiation towards the myeloid lineage are associated with aging HSCs $[4,5]$. Thus, HSC aging should contribute to the pathogenesis of many hematological diseases (such as hematological malignancies and bone marrow failure) and the efficacy of HSC transplantation. As an important note, caution must be taken on the specific phenotype or functional assay being used for HSCs when hematopoietic cells are analyzed under aging conditions.

In the current issue of International Journal of Hematology on "Progress in Hematology", we have invited several expert investigators to discuss some critical aspects surrounding HSC aging.

Chen J. provided an overview on HSC biology and a historic perspective on HSC aging studies. Especially, he summarized the work on HSC aging with different mouse strains, thus showing genetic factors as a critical basis for HSC aging. In addition, he also discussed the potential link of HSC aging with bone marrow failure diseases. Epigenetic factors are also important but have been less studied for HSC aging. Klauke K. and de Haan G. highlighted the current state of epigenetic regulation in HSCs and specifically, they discussed the important roles of Polycomb group (PcG) proteins as key epigenetic regulators of HSC cellular fate decisions and their link to human hematopoietic malignancies. Oxidative stress has long been thought to be associated with aging in general. There is no exception for HSC aging. Shao L. et al. reviewed the recent studies on the critical roles of reactive oxygen species (ROS) and its signaling events in HSCs in the contexts of HSC aging as well as radiation damage. As intrinsic cellular factors, telomere and cell cycle checkpoints are the 
ultimate cellular effectors in dealing with HSC aging. Ju Z. et al. discussed the current view on cell cycle control in HSCs and then focused on the role of telomere shortening and its regulatory mechanisms during HSC aging. Especially, the DNA damage response and its check point control were detailed in their review.

These reviews represent several important topics in the field of HSC aging, though some other important issues cannot be covered due to space limitation of this journal. While significant progress has been made during the last several years on the appreciation of the biological basis of HSC aging, it is far from the deeper understanding of the mechanisms and especially the link to the clinical problems. For example, how does the microenvironment cooperate with HSCs during aging? To what extent can HSC aging potentially be reversible? How does HSC aging contribute to disease process or vice versa? How does DNA damage response wire to multiple regulatory pathways in HSCs? These are critical issues in the field to be studied. Further studies in these areas will not only yield new medical intervention for hematological diseases (such as leukemia), but also benefit the studies on the aging of other stem cells and their applications.

\section{References}

1. Geiger H, Van Zant G. The aging of lympho-hematopoietic stem cells. Nat Immunol. 2002;3(4):329-33. (review).

2. de Haan G, Nijhof W, Van Zant G. Mouse strain-dependent changes in frequency and proliferation of hematopoietic stem cells during aging: correlation between lifespan and cycling activity. Blood. 1997;89(5):1543-50.

3. Harrison DE, Astle CM, Delaittre JA. Loss of proliferative capacity in immunohemopoietic stem cells caused by serial transplantation rather than aging. J Exp Med. 1978;147(5): 1526-31.

4. Morrison SJ, Wandycz AM, Akashi K, Globerson A, Weissman IL. The aging of hematopoietic stem cells. Nat Med. 1996;2(9): 1011-6.

5. Sudo K, Ema H, Morita Y, Nakauchi H. Age-associated characteristics of murine hematopoietic stem cells. J Exp Med. 2000; 192(9):1273-80. 